Z Herz- Thorax- Gefäßchir 2021 · 35:211-212 https://doi.org/10.1007/s00398-021-00433-2 Angenommen: 13. April 2021

Online publiziert: 26. April 2021

(c) Der/die Autor(en) 2021

\title{
Ludwig Müller
}

Univ.-Klinik für Herzchirurgie, Innsbruck, Österreich

\section{Chordae-Loops im Langzeitverlauf bewährt}

\section{Originalpublikation}

Pfannmueller B, Misfeld M, Verevkin A, Garbade J, Holzhey DM, Davierwala P, Seeburger J, Noack T, Borger MA (2021) Loop neochord versus leaflet resection techniques for minimally invasive mitral valve repair: long-term results. Eur J Cardiothorac Surg. 59(1) 180-186. https://doi.org/10.1093/ejcts/ezaa255.

Hintergrund ist die zunehmende Anwendung von künstlichen Sehnenfäden für die Rekonstruktion bei degenerativer Mitralklappeninsuffizienz als Alternative zur Segelresektion. Befeuert wurde die Diskussion um die beste Rekonstruktionsstrategie von Perier: „respect rather than resect“ [1]. Zur besseren Reproduzierbarkeit wurden Loops aus expandiertem Polytetrafluorethylen (ePTFE) von vorbestimmter Länge entwickelt [2] und weithin propagiert. Langzeitergebnisse fehlen bisher.

Patienten und Methoden. Bei 2134 konsekutiven Patienten mit Mitralklappenprolaps wurde von 1999 bis 2014 eine minimal-invasive Mitralklappenrekonstruktion über eine Minithorakotomie durchgeführt, bei 17,9\% mit Resektion, bei $82,1 \%$ mit ePTFE-Chordae-Loops.

Bei $86 \%$ der Patienten konnte ein Follow-up erfolgen, im Mittel 9,1 Jahre nach Resektion und 5,5 Jahre nach Loops. In den präoperativen Parametern unterschieden sich die Gruppen hinsichtlich kardialer Voroperationen $(1,1 \%$ in der Resektions-, 3,1\% in der Loop-Gruppe) und der LVEF (höherer Anteil von EF $<60 \%$ in der Resektionsgruppe).

Ergebnisse. Loops-Patienten hatten längere Klemm- und Bypass-Zeiten, mehr simultane Eingriffe (TKR, ASD/PFO) und erhielten einen größeren Anulo- plastiering (im Mittel 32,8 vs. 30,9 mm). Die 30-Tage-Mortalität betrug $0,8 \%$, ohne statistisch signifikanten Unterschied zwischen den Gruppen.

Nach 10 Jahren waren Gesamtüberleben und Freiheit von kardialem Tod signifikant besser in der Loops-Gruppe; die Reoperationsraten vergleichbar.

Kommentar. Die dargestellten Ergebnisse der Loops-Technik sind ein überaus wichtiger Beitrag und unterstützen ihre weitverbreitete Anwendung. Kritikern der Methode bei „excessive tissue“ wird durch die überlegenen oder äquivalenten Langzeitergebnisse ein gewichtiges Argument abhandenkommen. Auch die eigenen Ergebnisse bestätigen die Leipziger Ergebnisse [3].

Limitationen (neben den von den $\mathrm{Au}$ toren erwähnten):

Das Barlow-Syndrom wird nicht erwähnt. Relevante pathophysiologische Erkenntnisse wie funktioneller Prolaps und „mitral annular disjunction“ wurden in den letzten Jahren in die chirurgische Evaluation und Behandlungsstrategie integriert. Dieselbe Arbeitsgruppe hat 2015 den Vergleich von minimal-invasiver Loop-Technik und Alfieri-Plastik bei Barlow-Syndrom publiziert [4]. Es ist unklar, wie diese Patienten in die vorliegende Analyse integriert sind, denn zweifellos handelt es sich dabei um einen degenerativen Mitralklappenprolaps und weder um eine Resektion noch um eine Neochordae-Implantation.

Anuloplastie: Abgesehen von den mittleren Ringgrößen fehlen alle Angaben (Prozentsatz, Art und Typen der Ringe). Eine Vielzahl ist am Markt und hat sich während der Studienzeit erheblich geändert. Dies könnte für den Langzeitverlauf bedeutsam sein. 
Konversionen $\mathrm{zu}$ Klappenersatz und medianer Sternotomie werden nicht berichtet, könnten aber die Ergebnisse beeinflusst haben.

$14 \%$ "lost to follow up“: Es muss befürchtet werden, dass mehr Patienten mit den Endpunkten Reoperation und Tod nicht im Follow-up zu finden sind als Patienten mit einem günstigen Verlauf.

Diskussion. Erstmalig werden Langzeitergebnisse der Loop-Technik in der minimal-invasiven Mitralklappenrekonstruktion in einer retrospektiven „Singlecenter"-Studie präsentiert.

Die vorliegende Studie unterstützt die „Respect-rather-than-resect“-Strategie weit über die ursprüngliche Empfehlung [1] hinaus.

Friedrich Mohr hat mit Entwicklung und Verbreitung dieser Technik einen unschätzbaren Beitrag zur erfolgreichen Mitralklappenrekonstruktion geleistet, insbesondere im minimal-invasiven, voll endoskopischen Setting, sowie für das Verständnis der Pathophysiologie der Mitralklappe. Dafür gebührt ihm allerhöchste Anerkennung.

\section{Fazit für die Praxis}

Die dargestellten Ergebnisse der minimal-invasiven Mitralklappenrekonstruktion mit der Loop-Neochordae-Technik sind ein Meilenstein und sollten für die Wahl der Rekonstruktionsstrategie berücksichtigt werden.

\section{Korrespondenzadresse}

Ao. Univ.-Prof. Dr.
Ludwig Müller
Univ.-Klinik für Herzchirurgie
6020 Innsbruck, Österreich
ludwig.mueller@
tirol-kliniken.at

Funding. Open access funding provided by University of Innsbruck and Medical University of Innsbruck.

Interessenkonflikt. L. Müller hat entgeltliche Beratertätigkeiten, Vorträge und Proctoring von minimalinvasiven Mitralklappenoperationen für Edwards Life Sciences, Medtronic, Abbott, Serag-Wiessner und Aesculap durchgeführt.

Open Access. Dieser Artikel wird unter der Creative Commons Namensnennung 4.0 International Lizenz veröffentlicht, welche die Nutzung, Vervielfältigung,
Bearbeitung, Verbreitung und Wiedergabe in jeglichem Medium und Format erlaubt, sofern Sie den/die ursprünglichen Autor(en) und die Quelle ordnungsgemäß nennen, einen Link zur Creative Commons Lizenz beifügen und angeben, ob Änderungen vorgenommen wurden.

Die in diesem Artikel enthaltenen Bilder und sonstiges Drittmaterial unterliegen ebenfalls der genannten Creative Commons Lizenz, sofern sich aus der Abbildungslegende nichts anderes ergibt. Sofern das betreffende Material nicht unter der genannten Creative Commons Lizenz steht und die betreffende Handlung nicht nach gesetzlichen Vorschriften erlaubt ist, ist für die oben aufgeführten Weiterverwendungen des $\mathrm{Ma}$ terials die Einwilligung des jeweiligen Rechteinhabers einzuholen.

Weitere Details zur Lizenz entnehmen Sie bitte der Lizenzinformation auf http://creativecommons.org/ licenses/by/4.0/deed.de.

\section{Literatur}

1. Perier $P$ et al (2008) Toward a new paradigm for the reconstruction of posterior leaflet prolapse: midterm results of the "respect rather than resect" approach. Ann Thorac Surg 86:718-725

2. Von Oppell UO, Mohr FW (2000) Chordal replacement for both minimally invasive and conventional mitral valve surgery using premeasured Gore-Tex loops. Ann Thorac Surg 70(6):2166-2168

3. Bonaros N et al (2020) Predictors of safety and success in minimally invasive surgery for degenerative mitral disease. EACTS 34th Annual Meeting.

4. Da Rocha e Silva JG etal (2015) Barlow's mitral valve disease: a comparison of neochordal (loop) and edge-to-edge (Alfieri) minimally invasive repair techniques. Ann Thorac Surg 100:2127-2135

\section{Genetik von Lungenkrankheiten}

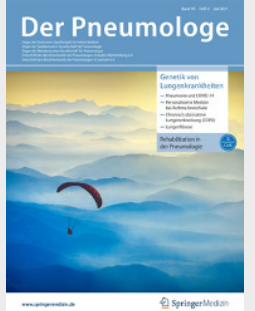

Erkrankungen der Atemwege und des Lungenparenchyms gehören weltweit zu den wichtigsten Erkrankungen hinsichtlich Prävalenz, Morbidität

und Mortalität. Oft konzentrieren sich klinische und pathophysiologische Betrachtungen auf exogene Auslöser, wie z.B. Infektionserreger, Allergene oder toxische Substanzen. Jedoch steht einer oftmals großen Gruppe von Exponierten nur eine begrenzte Zahl tatsächlich Erkrankter gegenüber. Dies legt einen bedeutsamen Einfluss endogener, unter anderem genetischer Ursachen nahe.

In Der Pneumologe 04/2021 stellen klinisch und grundlagenwissenschaftlich erfahrene Kolleg*innen die Bedeutung genetischer Ursachen für wichtige Lungenkrankheiten dar und beschreiben die Implikationen für die Behandlung der Erkrankten.

- Bedeutung der Genetik für die Pneumonie und COVID-19

- Genetik und personalisierte Medizin bei Asthma bronchiale

- Genetische Ursachen der chronisch obstruktiven Lungenerkrankung (COPD)

- Genetik der Lungenfibrose

Suchen Sie noch mehr zum Thema? Mit e.Med - den maßgeschneiderten Fortbildungsabos von Springer Medizin - haben Sie Zugriff auf alle Inhalte von SpringerMedizin.de. Sie können schnell und komfortabel in den für Sie relevanten Zeitschriften recherchieren und auf alle Inhalte im Volltext zugreifen.

Weitere Infos zu e.Med finden Sie auf springermedizin.de unter „Abos" 